\title{
ON ASYMPTOTIC NORMALITY OF THE HILL ESTIMATOR
}

\author{
Laurens de Haan and Sidney Resnick \\ Erasmus University Rotterdam \\ Cornell University
}

\begin{abstract}
For iid observations $X_{1}, \ldots, X_{n}$ from a common distribution $F$ with regularly varying tail $1-F(x) \sim$ $x^{-\alpha} L(x), x \rightarrow \infty$, the most popular estimator of $\alpha$ is the Hill estimator. Regular variation of the distribution tail is equivalent to weak consistency of the Hill estimator in a manner made precise in Mason (1983) but necessary and sufficient conditions for asymptotic normality of this estimator are still somewhat shrouded in confusion. This is in part due to the different possibilities for a centering in the asymptotic normality statement. We clarify the roles played by smoothness conditions such as Von Mises conditions for the asymptotic normality and give a minimal condition under which a non constant centering can be used.
\end{abstract}

\section{Introduction.}

Consider independent, identically distributed random variables $\left\{X_{n}, n \geq 1\right\}$ with common distribution $F$ with a regularly varying tail

$$
P\left[X_{1}>x\right]=x^{-\alpha} L(x), \quad x \rightarrow \infty, \alpha>0
$$

where $L$ is a slowly varying function and $\alpha$ is the index of regular variation. Set $\gamma=1 / \alpha$. Such distributions have been increasingly important as large data sets from telecommunications (file lengths, call holding times, transmission times, packet interarrival times) and finance (returns, exchange rates) have been found to exhibit heavy tail characteristics.

The goal is to estimate $\alpha$ or equivalently $\gamma$ and the most popular estimator for this purpose has been the Hill estimator $H_{k, n}$ defined as follows: For a random sample $X_{1}, \ldots, X_{n}$ of size $n$, let

$$
X_{(1)} \geq X_{(2)} \geq \cdots \geq X_{(n)}
$$

be the order statistics in decreasing order. Then the Hill estimator $H_{k, n}$ based on $k+1$ upper order statistics and a sample of size $n$ is

$$
H_{k, n}=\frac{1}{k} \sum_{i=1}^{k} \log \frac{X_{(i)}}{X_{(k+1)}} .
$$

Concerning asymptotic behavior of this estimator, it is known that $H_{k, n}$ is consistent in the sense that

$$
H_{k, n} \stackrel{P}{\rightarrow} \gamma
$$

Key words and phrases. regular variation, second order behavior, tail empirical measure, extreme value theory, maxima, Hill estimator, asymptotic normality.

The research by L. de Haan and S. Resnick was partially supported by Nato Collaborative Research Grant CRG 901020 and S. Resnick received some support from the Tinbergen Institute of Erasmus University Rotterdam. S. Resnick gratefully acknowledges the hospitality of Erasmus University. S. Resnick was also partially supported by NSF Grant DMS-9400535 at Cornell University. 
provided $n \rightarrow \infty, k \rightarrow \infty$ and $n / k \rightarrow \infty$ as was proved by Mason (1983) and in fact Mason proved that in a certain sense consistency of the Hill estimator is equivalent to regular variation of $1-F$. Various authors have considered asymptotic normality of Hill's estimator and it is known that if $1-F$ satisfies a second order refinement of (1.1) frequently phrased as a second order regular variation condition then

$$
\sqrt{k}\left(H_{k, n}-\gamma\right) \Rightarrow N\left(0, \gamma^{2}\right)
$$

provided $n \rightarrow \infty, k \rightarrow \infty, n / k \rightarrow \infty$ and an additional restriction on the sequence $\{k(n)\}$ depending on the second order condition is satisfied. See for example Hausler and Teugels (1985), Csorgo and Mason (1985), Davis and Resnick (1984), Goldie and Smith (1987). Geluk de Haan, Resnick and Stărică (1995) have shown that under a strengthening of (1.1) called the Von Mises conditions, second order regular variation is equivalent to asymptotic normality of the Hill estimator. The Von Mises condition requires $F$ have a density $F^{\prime}$ near $\infty$ which satisfies

$$
\frac{x F^{\prime}(x)}{1-F(x)} \rightarrow \alpha, \quad(x \rightarrow \infty)
$$

We became curious about why it was necessary to suppose the Von Mises condition held, what role this condition played in the asymptotic normality of the Hill estimator and if the condition could be weakened. We also sought to clarify the different conditions sufficient for (1.2) and for the slightly weaker statement

$$
\sqrt{k}\left(H_{k, n}-\gamma_{k, n}\right) \Rightarrow N\left(0, \gamma^{2}\right)
$$

for some sequence of constants $\left\{\gamma_{k, n}\right\}$.

Section 2 gives a sequential condition on the inverse of the distribution tail which is equivalent to asymptotic normality of $H_{k, n}$ given in (1.4). Section 3 discusses this sequential condition in more detail and gives several characterizations of distributions satisfying this condition. Concluding remarks and a summary are given in Section 4.

\section{Asymptotic normality of the Hill estimator.}

Our starting point is the following basic random measure result.

Proposition 2.1. Suppose $\left\{X_{n}, n \geq 1\right\}$ are iid non-negative random variables with common distribution $F$ whose tail is regularly varying so that (1.1) holds. Let $b(t)$ be the quantile function defined by

$$
b(t)=\left(\frac{1}{1-F}\right)^{\leftarrow}(t) .
$$

Let the tail empirical measure be

$$
\nu_{n}(\cdot)=\frac{1}{k} \sum_{i=1}^{n} \epsilon_{X_{i} / b(n / k)}(\cdot)
$$

so that $k \nu_{n}(A)$ is the cardinality of $\left\{i \leq n: X_{i} / b(n / k) \in A\right\}$. Then if $k=k(n)$ satisfies $k \rightarrow \infty$ and $k / n \rightarrow 0$, we have

$$
\sqrt{k}\left(\nu_{n}((x, \infty])-E \nu_{n}((x, \infty])\right) \Rightarrow W\left(x^{-\alpha}\right)
$$

in $D((0, \infty])$, where $\{W(t), t \geq 0\}$ is a standard Brownian motion.

Note that

$$
\left.E \nu_{n}((x, \infty])\right)=\frac{n}{k}\left(1-F\left(b\left(\frac{n}{k}\right) x\right)\right)=\frac{n}{k}\left(\bar{F}\left(b\left(\frac{n}{k}\right) x\right)\right) .
$$


Here and throughout we follow the convention that $\bar{F}=1-F$. The proof of Proposition 2.1 is based on the Lindeberg-Feller central limit theorem and does not differ much from the classical proof in Billingsley (1968). See de Haan and Resnick (1993), Resnick and Stărică (1995), Mason (1988). The result is also used in Geluk de Haan, Resnick and Stărică (1995).

We now modify (2.1) by writing

$$
\sqrt{k}\left(\nu_{n}\left(\left(x^{-\gamma}, \infty\right]\right)-\frac{n}{k} \bar{F}\left(b\left(\frac{n}{k}\right) x^{-\gamma}\right)\right) \Rightarrow W(x)
$$

in $D[0, \infty)$ and letting

$$
V_{n}(x):=\frac{n}{k} \bar{F}\left(b\left(\frac{n}{k}\right) x^{-\gamma}\right)
$$

(so that $V_{n}(x) \rightarrow x$ and $V_{n}^{\leftarrow}(x) \rightarrow x$ locally uniformly as $n \rightarrow \infty$ ) we have

$$
\sqrt{k}\left(\nu_{n}\left(\left(V_{n}^{\leftarrow}(y)\right)^{-\gamma}, \infty\right]-y\right) \Rightarrow W(y)
$$

in $D[0, \infty)$. Applying Vervaat's lemma (Vervaat, 1972) we get by inversion

$$
\left.\sqrt{k}\left(\left(\nu_{n}\left(V_{n}^{\leftarrow}(y)\right)^{-\gamma}, \infty\right]\right)^{\leftarrow}-y\right) \Rightarrow-W(y)
$$

in $D[0, \infty)$. Evaluating the left side of $(2.4)$ yields

$$
\sqrt{k}\left(\frac{n}{k} \bar{F}\left(X_{([k x])}\right)-x\right) \Rightarrow-W(x)
$$

in $D[0, \infty)$ and in fact the convergence in $(2.1)$ and $(2.5)$ are joint in $D(0, \infty] \times D[0, \infty)$. Observe that $(2.5)$ implies that

$$
\frac{n}{k} \bar{F}\left(X_{([k x])}\right) \Rightarrow x
$$

in $D[0, \infty)$ and regular variation yields

$$
\frac{X_{([k x])}}{b(n / k)} \Rightarrow x^{\gamma}
$$

Note that (2.1) and (2.6) hold jointly. Set $x=1$ in (2.6) and apply the composition map

$$
(x(t), y(t)) \mapsto x(t y(t))
$$

to get

$$
\sqrt{k}\left(\frac{1}{k} \sum_{i=1}^{n} \epsilon_{X_{i} / X_{(k)}}(x, \infty]-\frac{n}{k} \bar{F}\left(X_{(k)} x\right)\right) \Rightarrow W\left(x^{-\alpha}\right)
$$

and now apply the map

$$
x \mapsto \int_{1}^{\infty} x(s) \frac{d s}{s}
$$

and after a truncation argument as in Resnick and Stărică (1995)) we get

$$
\sqrt{k}\left(H_{k, n}-\int_{X_{(k)}}^{\infty} \frac{n}{k} \bar{F}(s) \frac{d s}{s}\right) \Rightarrow \int_{1}^{\infty} W\left(x^{-\alpha}\right) \frac{d x}{x} .
$$


The convergence in (2.7) is a consequence of Proposition 2.1 and supposes only that $\bar{F}$ is regularly varying. We now attempt to replace this random centering $\int_{X_{(k)}}^{\infty} \frac{n}{k} \bar{F}(s) \frac{d s}{s}$ with a deterministic centering. Since $X_{(k)} / b(n / k) \stackrel{P}{\rightarrow} 1$ we hope we can use $\int_{b(n / k)}^{\infty} \frac{n}{k} \bar{F}(s) \frac{d s}{s}$ in place of the random centering. In order to replace the random centering by this deterministic one we thus need

$$
\sqrt{k}\left(\int_{X_{(k)}}^{\infty} \frac{n}{k} \bar{F}(s) \frac{d s}{s}-\int_{b(n / k)}^{\infty} \bar{F}(s) \frac{d s}{s}\right) \Rightarrow V,
$$

for some non-degenerate random variable $V$. If the Von Mises condition (1.3) holds, it is not hard to verify that (2.8) indeed holds since

$$
\begin{aligned}
\sqrt{k} \int_{X_{(k)}}^{b(n / k)} \frac{n}{k} \bar{F}(s) \frac{d s}{s} & \sim \frac{\sqrt{k}}{\alpha} \int_{X_{(k)}}^{b(n / k)} \frac{n}{k} F^{\prime}(s) d s \\
& =\frac{\sqrt{k}}{\alpha}\left(\frac{n}{k} \bar{F}\left(X_{(k)}\right)-\frac{n}{k} \bar{F}(b(n / k))\right) \\
& =\frac{\sqrt{k}}{\alpha}\left(\frac{n}{k} \bar{F}\left(X_{(k)}\right)-1\right) \\
& \Rightarrow-\frac{1}{\alpha} W(1),
\end{aligned}
$$

from $(2.5)$.

We summarize this discussion so far (Davis and Resnick, 1988; Mason, 1988).

Proposition 2.2. If $F$ has a regularly varying tail as in (1.1) and the Von Mises condition (1.3) holds, we have

$$
\sqrt{k}\left(\int_{X_{(k)}}^{\infty} \frac{n}{k} \bar{F}(s) \frac{d s}{s}-\int_{b(n / k)}^{\infty} \frac{n}{k} \bar{F}(s) \frac{d s}{s}\right) \Rightarrow-\frac{1}{\alpha} W(1),
$$

and

$$
\sqrt{k}\left(H_{k, n}-\int_{b(n / k)}^{\infty} \frac{n}{k} \bar{F}(s) \frac{d s}{s}\right) \Rightarrow \int_{1}^{\infty} W\left(s^{-\alpha}\right) \frac{d s}{s}-\frac{1}{\alpha} W(1)
$$

and thus $H_{k, n}$ is asymptotically normal with asymptotic mean $\int_{b(n / k)}^{\infty} \frac{n}{k} \bar{F}(s) s^{-1} d s$ and variance $\gamma^{2}=1 / \alpha^{2}$.

Proof. The result follows because (2.9) holds jointly with (2.7). The variance of the limit can be calculated easily from the covariance function of Brownian motion.

Remark. In the presence of the regular variation condition (1.1) and the Von Mises condition (1.3), the function of a second order regular variation condition is to allow the replacement of $\int_{b(n / k)}^{\infty} \frac{n}{k} \bar{F}(s) s^{-1} d s$ by the centering $\gamma$. See for example Geluk, de Haan, Resnick and Stărică(1995).

We now seek minimal conditions for (2.8) which replace the Von Mises condition. For this purpose set

$$
U(t)=b(t)=\left(\frac{1}{1-F}\right)^{\leftarrow}(t)
$$

and rewrite $(2.8)$ as

$$
\sqrt{k} \int_{U^{\leftarrow}\left(X_{(k)}\right)}^{n / k} \frac{n}{k} s^{-1} d \log U(s) \Rightarrow V
$$


From (2.5) and the delta method we get

$$
\sqrt{k}\left(\frac{1}{\frac{n}{k} \bar{F}\left(X_{(k)}\right)}-1\right) \Rightarrow W(1)
$$

or equivalently

$$
W_{k}:=\sqrt{k}\left(\frac{U^{\leftarrow}\left(X_{(k)}\right)}{n / k}-1\right) \Rightarrow W(1) .
$$

Observe that

$$
U^{\leftarrow}\left(X_{(k)}\right)=\frac{n}{k}\left(\frac{W_{k}}{\sqrt{k}}+1\right) .
$$

and thus (2.11) can be rewritten as

$$
\sqrt{k} \int_{n / k}^{\frac{n}{k}\left(1+W_{k} / \sqrt{k}\right)} \frac{n}{k} s^{-1} d \log U(s) \Rightarrow-V .
$$

Define the nondecreasing, right continuous function

$$
\chi_{n}(x)=\sqrt{k} \int_{n / k}^{\frac{n}{k}(1+x / \sqrt{k})} \frac{n}{k} s^{-1} d \log U(s)
$$

and then at continuity points of the limit

$$
P\left[\chi_{n}\left(W_{k}\right) \leq x\right]=P\left[W_{k} \leq \chi_{n}^{\leftarrow}(x)\right] \rightarrow P[-V \leq x]
$$

and since $W_{k} \Rightarrow W(1)$ we get there must exist a nondecreasing function $\chi_{\infty}(x)$ with more than one point of increase such that that

$$
\chi_{n}^{\leftarrow}(x) \stackrel{w}{\rightarrow} \chi_{\infty}^{\leftarrow}(x) \quad \text { and } \quad \chi_{n}(x) \stackrel{w}{\rightarrow} \chi_{\infty}(x)
$$

and with $N(x)$ denoting the standard normal distribution function, we have

$$
N\left(\chi_{\infty}^{\leftarrow}(x)\right)=P[-V \leq x] \quad \text { and } \quad-V \stackrel{d}{=} \chi_{\infty}(W(1)) .
$$

Since for $x>0$

$$
\begin{aligned}
\chi_{\infty}(x) & \leq \liminf _{n \rightarrow \infty} \frac{\log U\left(n k^{-1}(1+x / \sqrt{k})\right)-\log U\left(n k^{-1}\right)}{(1+x / \sqrt{k}) 1 / \sqrt{k}} \\
& \leq \limsup _{n \rightarrow \infty} \frac{\log U\left(n k^{-1}(1+x / \sqrt{k})\right)-\log U\left(n k^{-1}\right)}{1 / \sqrt{k}} \\
& \leq \chi_{\infty}(x)
\end{aligned}
$$

with similar inequalities holding for $x<0$, we have as a consequence of $1+x k^{-1 / 2} \sim 1$ that (2.13) is equivalent to

$$
\lim _{n \rightarrow \infty} \frac{\log U\left(n k^{-1}(1+x / \sqrt{k})\right)-\log U\left(n k^{-1}\right)}{1 / \sqrt{k}}=\chi_{\infty}(x) .
$$

We summarize. 
Proposition 2.3. For a sequence $k$ satisfying $k \rightarrow \infty$ and $k / n \rightarrow 0$ we have (2.8) holding with a nondegenerate limit random variable $V$ iff there exists a nondecreasing function $\chi_{\infty}$ with more than one point of increase such that

$$
\lim _{n \rightarrow \infty} \frac{\log U\left(n k^{-1}(1+x / \sqrt{k})\right)-\log U\left(n k^{-1}\right)}{1 / \sqrt{k}}=\chi_{\infty}(x)
$$

weakly, in which case

$$
N\left(\chi_{\infty}^{\leftarrow}(x)\right)=P[-V \leq x] \quad \text { and } \quad-V \stackrel{d}{=} \chi_{\infty}(W(1))
$$

and

$$
\sqrt{k}\left(H_{k, n}-\int_{b(n / k)}^{\infty} \frac{n}{k} \bar{F}(s) \frac{d s}{s}\right) \Rightarrow \int_{1}^{\infty} W\left(x^{-\alpha}\right) \frac{d x}{x}-\chi_{\infty}(W(1)) .
$$

Furthermore, there exist constants $\gamma_{k, n}$ and a normal random variable $N$ such that

$$
\sqrt{k}\left(H_{k, n}-\gamma_{k, n}\right) \Rightarrow N
$$

iff (2.8) and hence (2.15) hold in which case

$$
\sqrt{k}\left(\int_{b(n / k)}^{\infty} \frac{n}{k} \bar{F}(s) \frac{d s}{s}-\gamma_{k, n}\right) \rightarrow c
$$

for some $c \in \mathbb{R}$ and

$$
\sqrt{k}\left(H_{k, n}-\int_{b(n / k)}^{\infty} \frac{n}{k} \bar{F}(s) \frac{d s}{s}\right) \Rightarrow \int_{1}^{\infty} W\left(x^{-\alpha}\right) \frac{d x}{x}-\chi_{\infty}(W(1))+c .
$$

Proof. Given (2.17), write

$$
\begin{aligned}
\sqrt{k}\left(H_{k, n}-\gamma_{k, n}\right)= & \sqrt{k}\left(H_{k, n}-\int_{X_{(k)}}^{\infty} \frac{n}{k} \bar{F}(s) \frac{d s}{s}\right)+\sqrt{k}\left(\int_{X_{(k)}}^{\infty} \frac{n}{k} \bar{F}(s) \frac{d s}{s}-\int_{b(n / k)}^{\infty} \frac{n}{k} \bar{F}(s) \frac{d s}{s}\right) \\
& +\sqrt{k}\left(\int_{b(n / k)}^{\infty} \frac{n}{k} \bar{F}(s) \frac{d s}{s}-\gamma_{k, n}\right) \\
= & \Theta_{n}+\chi_{n}\left(W_{k}\right)+\delta_{n} \\
= & \Theta_{n}+\psi_{n}\left(W_{k}\right),
\end{aligned}
$$

where

$$
\delta_{n}=\sqrt{k}\left(\int_{b(n / k)}^{\infty} \frac{n}{k} \bar{F}(s) \frac{d s}{s}-\gamma_{k, n}\right)
$$

and

$$
\psi_{n}(x)=\chi_{n}(x)+\delta_{n}=\sqrt{k} \int_{n / k}^{n k^{-1}(1+x / \sqrt{k})} \frac{n}{k} s^{-1} d \log U(s)+\delta_{n} .
$$


The function $\psi_{n}(\cdot)$ is nondecreasing. We know that first of all

$$
\Theta_{n}+\psi_{n}\left(W_{k(n)}\right) \Rightarrow N
$$

in $\mathbb{R}$ and secondly that

$$
\left(\Theta_{n}, W_{k(n)}\right) \Rightarrow\left(N_{1}, N_{2}\right)
$$

in $\mathbb{R}^{2}$ where $\left(N_{1}, N_{2}\right)$ is a normal random vector. By the selection theorem, $\psi_{n}$ has weak subsequential limits. If two subsequential limits were different, $(2.19)$ and $(2.20)$ would be contradicted. Thus $\psi_{n} \stackrel{w}{\rightarrow} \psi_{\infty}$. The function $\psi_{\infty}(x)$ must be finite for all $x$; otherwise, $N$ would not be $\mathbb{R}$-valued. Also $\psi_{\infty}$ must be continuous since otherwise $N_{1}+\psi_{\infty}\left(N_{2}\right) \stackrel{d}{=} N$ would not have a continuous distribution. Thus 0 is in the continuity set of $\psi_{\infty}$ and

$$
\psi_{n}(0)=\delta_{n} \rightarrow \psi_{\infty}(0)
$$

and therefore

$$
\chi_{n}(x) \stackrel{w}{\rightarrow} \chi_{\infty}(x)=\psi_{\infty}(x)-\psi_{\infty}(0)
$$

The next section contains a more detailed discussion of (2.15). Here we note two elementary facts. First, observe that if the Von Mises condition (1.3) holds then

$$
\lim _{x \rightarrow \infty} \frac{x U^{\prime}(x)}{U(x)}=\gamma
$$

and so (2.15) becomes

$$
\begin{aligned}
\frac{\log U\left(n k^{-1}(1+x / \sqrt{k})\right)-\log U\left(n k^{-1}\right)}{1 / \sqrt{k}} & =\sqrt{k} \int_{n / k}^{n k^{-1}(1+x / \sqrt{k})} \frac{s U^{\prime}(s)}{U(s)} \frac{d s}{s} \\
& \sim \gamma \sqrt{k} \int_{n / k}^{n k^{-1}(1+x / \sqrt{k})} \frac{d s}{s} \\
& =\gamma \sqrt{k} \log (1+x / \sqrt{k}) \sim \gamma \sqrt{k} \frac{x}{\sqrt{k}} \\
& =\gamma x
\end{aligned}
$$

and so (2.16) yields a limit of $\int_{1}^{\infty} W\left(x^{-\alpha}\right) \frac{d x}{x}-\gamma W(1)$. We will see in Section 3 that under very mild restrictions on $\{k(n)\}$, the limit in $(2.15)$ has to be $\chi_{\infty}(x)=\gamma x$. Second, we note that in inverted form, (2.15) seems to be a kind of local second order condition:

$$
\sqrt{k}\left(1-\frac{n}{k} \bar{F}\left(b\left(\frac{n}{k}\right)\left(1+\frac{y}{\sqrt{k}}\right)\right)\right) \stackrel{w}{\rightarrow} \chi_{\infty}^{\leftarrow}(y) .
$$

\section{Analytic condition.}

We now explore in more depth the significance of the condition (2.15). To make progress in analyzing (2.15) it is necessary to impose a mild restriction on the sequence $k(n)$ and in this section we suppose the sequence $k(n)$ is related to a self neglecting (SN) function. An eventually positive function $a(t)$ is called self neglecting (written $a(\cdot) \in S N$ ) if

$$
\lim _{t \rightarrow \infty} \frac{a(t+x a(t))}{a(t)}=1
$$


locally uniformly in $x \in \mathbb{R}$. A convenient sufficient condition for a function $a(\cdot)$ to be SN is that the derivative $a^{\prime}(\cdot)$ exist and $\lim _{t \rightarrow \infty} a^{\prime}(t)=0$. See for instance Bingham, Goldie and Teugels, (1987); Geluk and de Haan (1987), Section I.4. Throughout, we will also assume that

$$
A(t):=\int_{0}^{t} \frac{1}{a(s)} d s
$$

is finite for all $t$.

We now describe the way the function $k(\cdot)$ needs to be related to a self neglecting function. Since $n / k \rightarrow \infty$, we set $\eta(n)=n / k$ and suppose that $\eta(\cdot)$ is nondecreasing and there is a SN function $a(\cdot)$ such that $a(t) \rightarrow 0$ and

$$
\eta^{\leftarrow}(s)=s / a^{2}(\log s)
$$

This is not very restrictive. For instance, if $k(n)=n^{\theta}$, for some $0<\theta<1$, then $\eta^{\leftarrow}(s)=s^{1 /(1-\theta)}$ and we find

$$
a(u)=\exp \left\{-\frac{\theta / 2}{1-\theta} u\right\}
$$

which is SN since $a^{\prime}(u) \rightarrow 0$ as $u \rightarrow \infty$. More generally, if $k(\cdot)$ is regularly varying with some index $\delta \in[0,1)$ (written $k(\cdot) \in R V_{\delta}$ ) then $\eta(\cdot) \in R V_{1-\delta}$ and $\eta^{\leftarrow} \in R V_{1 /(1-\delta)}$ and thus

$$
a \circ \log \in R V_{-\delta /(2(1-\delta))} \text {. }
$$

Note $a(\log s) \rightarrow 0$ and

$$
\begin{aligned}
\lim _{t \rightarrow \infty} \frac{a(t+x a(t))}{a(t)} & =\lim _{t \rightarrow \infty} \frac{a\left(\log e^{t+x a(t)}\right)}{a\left(\log e^{t}\right)} \\
& =\lim _{s \rightarrow \infty} \frac{a\left(\log s e^{x a(\log s)}\right)}{a(\log s)} \\
& =1,
\end{aligned}
$$

locally uniformly by the regular variation of $a(\cdot)$.

We now suppose the limit in (2.15) is continuous so that convergence in (2.15) holds locally uniformly. Suppose (3.1) holds and set $n / k(n)=\eta(n)$ and

$$
\begin{aligned}
\frac{n}{k}\left(1+\frac{x}{\sqrt{k}}\right) & =\frac{n}{k}+\frac{n x}{k^{3 / 2}} \\
& =\eta(n)+\frac{\eta(n) x}{\sqrt{n / \eta(n)}} \\
& =\eta(n)(1+x \sqrt{\eta(n) / n}) .
\end{aligned}
$$

Define the function

$$
V=\log \circ U \circ \exp
$$

and the local uniformity in (2.15) implies

$$
\frac{V(\log \eta(n)+\sqrt{\eta(n) / n} x)-V(\log \eta(n))}{\sqrt{\eta(n) / n}} \rightarrow \chi_{\infty}(x) .
$$


We suppose we may switch from the discrete variable $n$ to the continuous variable $t$ and set $s=\log \eta(t)$. (This transition is non-trivial and we give more detail at the end of the section.) ¿From (3.1) we have

$$
a(s)=\sqrt{\eta(t) / t}
$$

and hence (2.15) becomes

$$
\frac{V(s+a(s) x)-V(s)}{a(s)} \rightarrow \chi_{\infty}(x)
$$

locally uniformly in $x$. Section 3 is devoted to studying the limit relation (3.2). This relation appears in a study of Tauberian theorems by Bingham (1981), Bingham and Goldie $(1983,1988)$.

We first identify the limit in (3.2).

Proposition 3.1. Suppose $a(\cdot) \in S N$ and that $A(t)=\int_{0}^{t} 1 / a(s) d s<\infty$ for all $t$ and that (3.2) holds for $V$ a nondecreasing function. Then for some $c>0$ we have

$$
\chi_{\infty}(x)=c x .
$$

In addition, if $a(t) \rightarrow 0, V=\log \circ U \circ \exp$ and $U \in R V_{\gamma}, \gamma>0$, then $c=\gamma$.

Proof. As in de Haan (1974) or Bingham and Goldie (1983) or Bingham, Goldie and Teugels (1987, page 126) $A(t)=\int_{0}^{t} \frac{1}{a(s)} d s$ has the following properties:

(1) $A(t) \rightarrow \infty$ as $t \rightarrow \infty$. This is true for any SN function $a(\cdot)$ since $a(\cdot) \in S N$ implies $a(t) / t \rightarrow 0$.

(2) $A$ has the variational property

$$
\lim _{t \rightarrow \infty} A(t+x a(t))-A(t)=x,
$$

locally uniformly, or equivalently by inversion

$$
\lim _{s \rightarrow \infty} \frac{A^{\leftarrow}(s+y)-A^{\leftarrow}(s)}{a \circ A^{\leftarrow}(s)}=y,
$$

locally uniformly. We can also write (3.5) as

$$
\lim _{t \rightarrow \infty} \frac{A^{\leftarrow}(\log t y)-A^{\leftarrow}(\log t)}{a \circ A^{\leftarrow}(\log t)}=\log y,
$$

so that $A^{\leftarrow} \circ \log$ is in the function class $\Pi$ (Geluk and de Haan, 1987) with auxiliary function

$$
g:=a \circ A^{\leftarrow} \circ \log
$$

and hence $g \in R V_{0}$. Properties (3.4), (3.5) and $\left(3.5^{\prime}\right)$ follow from the fact that $a(\cdot) \in S N$ since

$$
\begin{aligned}
A(t+x a(t))-A(t) & =\int_{t}^{t+x a(t)} \frac{1}{a(s)} d s \\
& =\int_{0}^{x} \frac{a(t)}{a(t+s a(t))} d s \\
& \rightarrow x
\end{aligned}
$$

from the definition of a self neglecting function. 
Now consider the function

$$
H(x)=V \circ A^{\leftarrow}(x)
$$

and we claim that (3.2) implies that $H$ satisfies

$$
\lim _{t \rightarrow \infty} \frac{H(t+x)-H(t)}{a \circ A^{\leftarrow}(t)}=\chi_{\infty}(x) .
$$

To check we write

$$
\begin{aligned}
\lim _{t \rightarrow \infty} \frac{H(t+x)-H(t)}{\left.a \circ A^{\leftarrow}(t)\right)} & =\lim _{t \rightarrow \infty} \frac{V\left(A^{\leftarrow}(t+x)\right)-V\left(A^{\leftarrow}(t)\right)}{a \circ A^{\leftarrow}(t)} \\
& =\lim _{t \rightarrow \infty} \frac{V\left(A^{\leftarrow}(t+x)-A^{\leftarrow}(t)+A^{\leftarrow}(t)\right)-V\left(A^{\leftarrow}(t)\right)}{a \circ A^{\leftarrow}(t)}
\end{aligned}
$$

and applying (3.5) this is

$$
=\lim _{t \rightarrow \infty} \frac{V\left(a \circ A^{\leftarrow}(t)\left(x+\epsilon_{t}\right)+A^{\leftarrow}(t)\right)-V\left(A^{\leftarrow}(t)\right)}{a \circ A^{\leftarrow}(t)}
$$

where $\epsilon_{t} \rightarrow 0$ locally uniformly. Hence changing variables the limit is the same as

$$
\lim _{s \rightarrow \infty} \frac{V(s+x a(s))-V(s)}{a(s)}=\chi_{\infty}(x) .
$$

This proves (3.6).

We now modify (3.6) by the change of variables $t=\log s, x=\log y$ and $\operatorname{setting} h=H \circ \log$ and $g=a \circ A^{\leftarrow} \circ \log$ we get (3.6) in the form

$$
\lim _{s \rightarrow \infty} \frac{h(s y)-h(s)}{g(s)}=\chi_{\infty}(\log y)
$$

and it is well known (Geluk and de Haan, 1987) that the limit function must be of the form

$$
\chi_{\infty}(x)=c\left(\frac{e^{\rho x}-1}{\rho}\right)
$$

for some $\rho \in \mathbb{R}$. Since $g \in R V_{0}$, we must have $\rho=0$ and $\chi_{\infty}(x)=c x$. This proves (3.3).

Now suppose $a(t) \rightarrow 0, U \in R V_{\gamma}, \gamma>0$ and $V=\log \circ U \circ \exp$. We write (3.6) as

$$
\frac{H(\log t y)-H(\log t)}{a \circ A^{\leftarrow \circ} \circ \log (t)} \rightarrow c \log y
$$

so that $h=H \circ \log$ is in the function class $\Pi$ with auxiliary function $c a \circ A^{\leftarrow} \circ \log$. This means (Geluk and de Haan, 1987; Bingham and Goldie, 1982)) that there exists

$$
\rho(t) \sim c a \circ A^{\leftarrow \circ} \circ \log (t) \in R V_{0}
$$

and a constant $c_{1}$ such that

$$
h(t)=H(\log t)=\rho(t)+\int_{1}^{t} \frac{\rho(s)}{s} d s+c_{1} .
$$


Since $H \circ A=V$ we have from (3.9)

$$
\begin{aligned}
V(x) & =\rho(\exp \{A(x)\})+\int_{1}^{\exp \{A(x)\}} \frac{\rho(s)}{s} d s+c_{1} \\
& =\rho(\exp \{A(x)\})+\int_{c_{2}}^{x} \rho(\exp \{A(w)\}) \frac{d w}{a(w)}+c_{1} \\
& =V_{1}(x)+V_{2}(x),
\end{aligned}
$$

where $V_{1}=\rho \circ \exp \circ A$. Note

$$
\begin{aligned}
V_{2}^{\prime}(x) & =\rho(\exp \{A(x)\}) / a(x) \\
& \sim c a \circ A^{\leftarrow} \circ \log \circ \exp \circ A(x) / a(x) \sim c,
\end{aligned}
$$

that is,

$$
V_{2}^{\prime}(x) \rightarrow c .
$$

For $i=1,2$ define $U_{i}$ by

$$
V_{i}=\log \circ U_{i} \circ \exp
$$

Since $U \in R V_{\gamma}$

$$
\frac{U(t x)}{U(t)}=\exp \{V(\log t x)-V(\log t)\} \rightarrow x^{\gamma}, \quad x>0
$$

as $t \rightarrow \infty$. Since $V_{2}^{\prime}(x) \rightarrow c$ we have $U_{2} \in R V_{c}$ since $U_{2}$ satisfies the Von Mises condition for membership in $R V_{c}$. Since $U=U_{1} \cdot U_{2}$ we have

$$
\begin{aligned}
\frac{U(t x)}{U(t)} & =\frac{U_{1}(t x)}{U_{1}(t)} \frac{U_{2}(t x)}{U_{2}(t)} \sim x^{c} \frac{U_{1}(t x)}{U_{1}(t)} \\
& =x^{c} \exp \left\{V_{1}(\log t x)-V_{1}(\log t)\right\} \\
& =x^{c} \exp \left\{\rho\left(e^{A(t x)}\right)-\rho\left(e^{A(t)}\right)\right\} .
\end{aligned}
$$

Now

$$
\begin{aligned}
\rho\left(e^{A(t x)}\right) & \sim c a \circ A^{\leftarrow} \circ \log \circ \exp \circ A(t x) \\
& =c a(t x) \rightarrow 0
\end{aligned}
$$

for $x>0$ and hence (3.13) leads to the conclusion

$$
\frac{U(t x)}{U(t)} \rightarrow x^{c}
$$

Comparing (3.14) with (3.12) yields $c=\gamma$.

We now offer the following characterizations of (3.2). 
Theorem 3.2. Suppose $V$ is nondecreasing, $a(\cdot) \in S N, a(t) \rightarrow 0$, and $V=\log \circ U \circ \exp$ with $U \in R V_{\gamma}$, $\gamma>0$. Suppose $A(t)=\int_{0}^{t} 1 / a(s) d s$ is finite for all $t$. The following are equivalent.

(i) For any $x \in \mathbb{R}$,

$$
\lim _{t \rightarrow \infty} \frac{V(t+x a(t))-V(t)}{a(t)}=\gamma x
$$

(ii) $V$ has the representation

$$
V=H \circ A
$$

where $h=H \circ \log \in \Pi$ with auxiliary function

$$
\gamma a \circ A^{\leftarrow} \circ \log .
$$

(iii) $V$ satisfies

$$
\lim _{t \rightarrow \infty} \frac{V(t)-\exp \{-A(t)\} \int_{0}^{t} V(s) \exp \{A(s)\} \frac{d s}{a(s)}}{a(t)}=\gamma
$$

(iv) $V$ has the representation

where $V_{2}$ is differentiable with derivative $V_{2}^{\prime}$ and

$$
V_{2}^{\prime}(s) \rightarrow \gamma, \text { and } V_{1}(x) \sim \gamma a(x) .
$$

Equivalently, $U$ has the product representation

$$
U=\exp \{\gamma(x) a(\log x)\} U_{2}(x)
$$

where $U_{2}$ is differentiable with derivative $U_{2}^{\prime}$ which satisfies the Von Mises condition

$$
\frac{x U_{2}^{\prime}(x)}{U_{2}(x)} \rightarrow \gamma
$$

and $\gamma(x) \rightarrow \gamma$

(v) $V$ has the representation

$$
V=V_{3}+V_{4}
$$

where $V_{4}$ is differentiable with derivative $V_{4}^{\prime}$ and $V_{4}^{\prime}(x) \rightarrow \gamma$ and $V_{3}(x)=o(a(x))$. Equivalently, $U$ has the product representation

$$
U=\exp \{\epsilon(x) a(\log x)\} U_{4}
$$

where $U_{4}$ is differentiable with derivative $U_{4}^{\prime}$ satisfying the Von Mises condition (3.22) and $\epsilon(x) \rightarrow 0$. 
Proof. The equivalence of (i) and (ii) is covered in Proposition 3.1 and in Bingham and Goldie (1988). Also from Proposition 3.1 we have $h=H \circ \log =V \circ A^{\leftarrow} \circ \log \in \Pi$ with auxiliary function $\gamma a \circ A^{\leftarrow} \circ \log$ and this is equivalent to

$$
\begin{aligned}
1 & =\lim _{t \rightarrow \infty} \frac{h(t)-t^{-1} \int_{1}^{t} h(u) d u}{\gamma a \circ A^{\leftarrow} \circ \log (t)} \\
& =\lim _{t \rightarrow \infty} \frac{V \circ A^{\leftarrow} \circ \log (t)-t^{-1} \int_{1}^{t} V \circ A^{\leftarrow} \circ \log (u) d u}{\gamma a \circ A^{\leftarrow} \circ \log (t)} \\
& =\lim _{s \rightarrow \infty} \frac{V(s)-\exp \{-A(s)\} \int_{1}^{\exp \{A(s)\}} V \circ A^{\leftarrow} \log (u) d u}{\gamma a(s)} \\
& =\lim _{s \rightarrow \infty} \frac{V(s)-\exp \{-A(s)\} \int_{0}^{s} V(s) \exp \{A(s)\} \frac{d s}{a(s)}}{\gamma a(s)} .
\end{aligned}
$$

This shows the equivalence of (i), (ii), (iii).

The proof that (3.19) follows from (3.15) was given as part of Proposition 3.1. To see that (3.19) implies (3.15), note that $V_{2}$ satisfies (3.15) by the argument given after (2.16) and $V_{1}$ satisfies

$$
\begin{aligned}
\lim _{t \rightarrow \infty} \frac{V_{1}(t+x a(t))-V_{1}(t)}{a(t)} & =\lim _{t \rightarrow \infty} \frac{V_{1}(t+x a(t))}{a(t+x a(t))} \frac{a(t+x a(t))}{a(t)}-\frac{V_{1}(t)}{a(t)} \\
& =\gamma 1-\gamma=0 .
\end{aligned}
$$

Condition (v) is discussed in Bingham and Goldie (1988).

The results of Theorem 3.2 can be re-expressed in terms of $V^{\leftarrow}$. Suppose $V=\log \circ U$ oexp and $U=(1 /(1-$ $F))^{\leftarrow} \in R V_{\gamma}$, where $1-F$ is a distribution tail. Then results in terms of $V^{\leftarrow}$ will give us characterizations in terms of $1-F$. Begin by observing that $V$ satisfies (3.15) iff $V^{\leftarrow}$ satisfies

$$
\frac{V^{\leftarrow}\left(t+x a \circ V^{\leftarrow}(t)\right)-V^{\leftarrow}(t)}{a \circ V^{\leftarrow}(t)} \rightarrow \gamma^{-1} x=\alpha x,
$$

locally uniformly in $x$ as $t \rightarrow \infty$. This is the same type of limit relation as (3.15) with $V^{\leftarrow}$ in place of $V$ and $a \circ V^{\leftarrow}$ in place of $a(\cdot)$. To make the parallel exact, we must check that $a(\cdot) \in S N$ implies $a \circ V^{\leftarrow}(\cdot) \in S N$. This is readily verified: We write

$$
\lim _{t \rightarrow \infty} \frac{a \circ V^{\leftarrow}\left(t+x a \circ V^{\leftarrow}(t)\right)}{a \circ V^{\leftarrow}(t)}=\lim _{t \rightarrow \infty} \frac{a\left(V^{\leftarrow}(t)+a\left(V^{\leftarrow}(t)\right)\left(\frac{V^{\leftarrow}\left(t+x a \circ V^{\leftarrow}(t)\right)-V^{\leftarrow}(t)}{a \circ V^{\leftarrow}(t)}\right)\right)}{a \circ V^{\leftarrow}(t)}
$$

and because (3.29) holds locally uniformly and because $(a(s+y a(s)) \sim a(s), s \rightarrow \infty$, locally uniformly in $y$, we get the above limit the same as

$$
=\lim _{s \rightarrow \infty} a\left(s+\gamma^{-1} x a(s)\right) / a(s)=1
$$

locally uniformly, showing that $a \circ V^{\leftarrow} \in S N$.

We may now apply Theorem 3.2 to $V^{\leftarrow}$ and the self neglecting function $a \circ V^{\leftarrow}$ yielding conditions in terms of $1-F$. We do not bother reformulating all parts of Theorem 3.2 in terms of $V^{\leftarrow}$ or $F$. 
Corollary 3.3. Suppose the conditions of Theorem 3.2 hold and in addition suppose $F$ is a distribution function satisfying $1-F(x) \sim x^{-\alpha} L(x), x \rightarrow \infty$ with $\alpha=\gamma^{-1}>0$. Set

$$
V=\log \circ U \circ \exp , \quad U=\left(\frac{1}{1-F}\right)^{\leftarrow}
$$

so that $U \in R V_{\gamma}$. Suppose further that $a(\cdot) \in S N$ and $\lim _{t \rightarrow \infty} a(t)=0$. The following are equivalent.

(i) $V^{\leftarrow}$ satisfies

$$
\frac{V^{\leftarrow}\left(t+x a \circ V^{\leftarrow}(t)\right)-V^{\leftarrow}(t)}{a \circ V^{\leftarrow}(t)} \rightarrow \alpha x,
$$

locally uniformly in $x \in \mathbb{R}$ as $t \rightarrow \infty$.

(ii) $F$ satisfies

$$
1-F(x)=e^{-\alpha(x) a \circ V^{\leftarrow}(\log x)}\left(1-F_{2}(x)\right)
$$

where $1-F_{2}$ is a Von Mises function and $F_{2}$ has a density $F_{2}^{\prime}$ satisfying

$$
\lim _{x \rightarrow \infty} \frac{x F_{2}^{\prime}(x)}{1-F_{2}(x)}=\alpha
$$

and $\alpha(x) \rightarrow \alpha$.

(iii) $F$ satisfies

$$
1-F(x)=e^{-\epsilon(x) a \circ V^{-}(\log x)}\left(1-F_{4}(x)\right)
$$

where $1-F_{4}$ is a Von Mises function and $F_{4}$ has a density $F_{4}^{\prime}$ satisfying

$$
\lim _{x \rightarrow \infty} \frac{x F_{4}^{\prime}(x)}{1-F_{4}(x)}=\alpha,
$$

and $\epsilon(x) \rightarrow 0$.

Proof. If (i) holds then we apply Theorem 3.2 (iv) to conclude

$$
V^{\leftarrow}=V_{1}^{\leftarrow}+V_{2}^{\leftarrow}
$$

where $V_{2}^{\leftarrow}$ is differentiable with derivative satisfying

$$
\left(V_{2}^{\leftarrow}\right)^{\prime}(x) \rightarrow \alpha, \quad x \rightarrow \infty
$$

and

$$
V_{1}^{\leftarrow}(x) \sim \alpha a \circ V^{\leftarrow}(x) .
$$

Define $U_{2}^{\leftarrow}$ and $1-F_{2}$ by

$$
V_{2}^{\leftarrow}=\log \circ U_{2}^{\leftarrow} \circ \exp \text { and } \frac{1}{1-F_{2}}=U_{2}^{\leftarrow}
$$

Then

$$
\begin{aligned}
1-F(x) & \sim \exp \left\{V_{1}^{\leftarrow}(\log x)+V_{2}^{\leftarrow}(\log x)\right\} \\
& =\exp \left\{V_{1}^{\leftarrow}(\log x)\right\}\left(1-F_{2}(x)\right) \\
& =e^{-\alpha(x) a \circ V^{\leftarrow}(\log x)}\left(1-F_{2}(x)\right) .
\end{aligned}
$$


We note that (3.30) is equivalent to

$$
\frac{s\left(\frac{1}{1-F_{2}}\right)^{\prime}(s)}{\frac{1}{1-F_{2}(s)}}=\frac{s F_{2}^{\prime}(s)}{\left(1-F_{2}(s)\right)^{2}}\left(1-F_{2}(s)\right)=\frac{s F_{2}^{\prime}(s)}{1-F_{2}(s)} \rightarrow \alpha,
$$

which shows that $1-F_{2}$ satisfies the Von Mises conditions.

The rest is similar.

We close this section by discussing what is involved in justifying a transition from the sequential limit relation (2.15) to the limit relation taken on a continuous variable in (3.2). (Obviously (3.2) implies (2.15).)

We start with the relation (see just prior to (3.2))

$$
\frac{V(\log \eta(n)+x \sqrt{\eta(n) / n})-V(\log \eta(n))}{\sqrt{\eta(n) / n}} \rightarrow \chi_{\infty}(x),
$$

as $n \rightarrow \infty$ where the limit $\chi_{\infty}$ is assumed continuous, and the convergence is therefore locally uniform. We investigate what conditions yield (3.2). Recall $V=\log \circ U \circ \exp , U \in R V_{\gamma}$ and $U$ is nondecreasing.

Note that if

$$
\frac{\log \eta(n+1)-\log \eta(n)}{\sqrt{\eta(n) / n}} \rightarrow 0 \text { and } \eta(n+1) \sim \eta(n)
$$

as $n \rightarrow \infty$, then (3.31) implies (3.2). The reason is that for any $t$

$$
\begin{aligned}
& \frac{V(\log \eta(t)+x \sqrt{\eta(t) / t})-V(\log \eta(t))}{\sqrt{\eta(t) / t}} \\
& \quad \geq \frac{V(\log \eta([t])+x \sqrt{[t] /([t]+1)} \sqrt{\eta([t]) /[t]})-V(\log \eta([t]))+V(\log \eta([t])-V(\log \eta([t]+1))}{\sqrt{\eta([t]) /[t]} \sqrt{\eta([t]+1) / \eta([t])}} \\
& \sim \chi_{\infty}(x)-\frac{V(\log \eta([t]+1))-V(\log \eta([t]))}{\sqrt{\eta([t]) /[t]}}
\end{aligned}
$$

where we have used (3.32). Now the second term on the right without the minus sign and replacing [t] by $n$ becomes

$$
\begin{aligned}
& \frac{V\left(\log \eta(n)+\sqrt{\eta(n) / n}\left(\frac{\log \eta(n+1)-\log \eta(n)}{\sqrt{\eta(n) / n}}\right)\right)-V(\log \eta(n))}{\eta(n) / n} \\
& \quad=\frac{V(\log \eta(n)+\epsilon(n) \sqrt{\eta(n) / n})-V(\log \eta(n))}{\eta(n) / n} \\
& \rightarrow \chi_{\infty}(0)=0
\end{aligned}
$$

where $\epsilon(n) \rightarrow 0$. A similar upper bound can be constructed and so we see that (3.32) is a sufficient condition for (3.31) to imply (3.2).

What conditions guarantee $(3.32)$ ? It is enough that $\eta(\cdot)$ be differentiable and

$$
\lim _{t \rightarrow \infty} \frac{\sqrt{t} \eta^{\prime}(t)}{(\eta(t))^{3 / 2}}=0
$$


We claim that (3.33) implies

$$
\lim _{n \rightarrow \infty} \frac{\eta(n+\theta)}{\eta(n)}=1
$$

locally uniformly in $\theta$. Note that (3.34) certainly implies the second asymptotic relation in (3.32) and for the first we have for some $\left|\theta_{n}\right| \leq 1$ that

$$
\begin{aligned}
\frac{\log \eta(n+1)-\log \eta(n)}{\sqrt{\eta(n) / n}}= & \frac{\eta^{\prime}\left(n+\theta_{n}\right) / \eta\left(n+\theta_{n}\right)}{\sqrt{\eta(n) / n}} \\
& \sim \frac{\left(n+\theta_{n}\right)^{1 / 2} \eta^{\prime}\left(n+\theta_{n}\right)}{\left(\eta\left(n+\theta_{n}\right)\right)^{3 / 2}} \\
& \rightarrow 0
\end{aligned}
$$

from (3.34) and (3.33). So the proof that (3.33) implies (3.32) will be complete if we verify that (3.33) implies (3.34).

Since

$$
\lim _{n \rightarrow \infty} \frac{\eta(n+\theta)}{\eta(n)}=\lim _{n \rightarrow \infty} \exp \left\{\int_{n}^{n+\theta}(\log \eta)^{\prime}(v) d v\right\},
$$

we have (3.34) following from $(\log \eta)^{\prime}(v) \rightarrow 0$ as $v \rightarrow \infty$. But

$$
(\log \eta)^{\prime}(t)=\frac{\eta^{\prime}(t)}{\eta(t)}
$$

and from (3.33)

$$
\lim _{t \rightarrow \infty} \frac{\eta^{\prime}(t)}{\eta(t)} \sqrt{\frac{t}{\eta(t)}}=0
$$

so it is enough to show $t / \eta(t) \rightarrow \infty$. However

$$
\begin{aligned}
\lim _{t \rightarrow \infty} \frac{t}{\eta(t)} & =\lim _{s \rightarrow \infty} \frac{\eta^{\leftarrow}(s)}{s} \\
& =\lim _{s \rightarrow \infty} \frac{s / a^{2}(\log s)}{s}=\lim _{u \rightarrow \infty} \frac{1}{a^{2}(u)}=\infty
\end{aligned}
$$

since $a(u) \rightarrow 0$.

So we conclude that if (3.33) holds, we may make the transition from the sequential limit (2.15) to the continuous limit (3.2).

It is possible to express (3.33) in terms of the $\mathrm{SN}$ function $a(\cdot)$. We have (3.33) equivalent to

$$
0 \geq \frac{d}{d t}\left(\frac{1}{\sqrt{\eta\left(t^{2}\right)}}\right)=-\frac{\eta^{\prime}\left(t^{2}\right) t}{\eta^{3 / 2}\left(t^{2}\right)} \rightarrow 0
$$

as $t \rightarrow \infty$. Hence the derivative of the inverse function of $1 / \sqrt{\eta\left(t^{2}\right)}$ tends to $-\infty$; that is,

$$
\frac{d}{d s} \sqrt{\eta^{\leftarrow}\left(1 / s^{2}\right)} \rightarrow-\infty \quad(s \downarrow 0),
$$


and changing variables $t=s^{-1} \rightarrow \infty$ we get

$$
t^{2} \frac{d}{d t} \sqrt{\eta^{\leftarrow}\left(t^{2}\right)} \rightarrow \infty \quad(t \rightarrow \infty)
$$

Using the definition of $a(\cdot)$

$$
\eta^{\leftarrow}(s)=s / a^{2}(\log s)
$$

we find that (3.33) is equivalent to

$$
\begin{aligned}
t^{2} \frac{d}{d t} \sqrt{\frac{t^{2}}{a^{2}\left(\log t^{2}\right)}} & =t^{2} \frac{d}{d t}\left(\frac{t}{a(2 \log t)}\right) \\
& =t^{2}\left(\frac{a(2 \log t)-t a^{\prime}(2 \log t) 2 / t}{a^{2}(2 \log t)}\right) \\
& =\frac{t^{2}}{a(2 \log t)}\left(1-\frac{2 a^{\prime}(2 \log t)}{a(2 \log t)}\right) \rightarrow \infty .
\end{aligned}
$$

So a sufficient condition for (3.33) is that the SN function $a(\cdot)$ satisfy $a^{\prime} \rightarrow 0, a \rightarrow 0$ and

$$
\limsup _{t \rightarrow \infty} \frac{a^{\prime}(t)}{a(t)}<\frac{1}{2}
$$

This would also be sufficient for the transition from the sequential condition (2.15) to the continuous relation (3.2).

4. Concluding remarks and summary. Mason (1982) showed that consistency of the Hill estimator is equivalent to regular variation of the underlying distribution tail. Asymptotic normality of the Hill estimator requires more than the assumption of a regularly varying tail. Regular variation only implies asymptotic normality of $H_{k, n}$ with a random centering. In order to have asymptotic normality with a non-random and non-constant asymptotic mean, the Von Mises condition for the distribution tail is stronger than necessary and Corollary 3.3 or Theorem 3.2 give the minimal conditions on the distribution. In order to be able to replace the non-constant asymptotic mean by a constant centering, a somewhat stronger assumption like second order regular variation (Geluk de Haan, Resnick and Stărică, 1995) is necessary.

\section{REFERENCES}

Billingsley, P., Convergence of Probability Measures, Wiley, New York, 1968.

Bingham, N.H., Tauberian theorems for Borel and related summability methods, Preprint, University of London (1981).

Bingham, N.H. and Goldie, C.M., Extensions of regular variation. I: Uniformity and quantifiers. II: Representations and indices, Proc. London Math Soc 44 (1982), 473-496, 497-534.

Bingham, N.H. and Goldie, C.M., On one-sided Tauberian conditions, Analysis 3 (1983), 159-188.

Bingham, N.H. and Goldie, C.M., Riesz means and self-neglecting functions, Mathematische Zeitschrift 199 (1988), 443-454.

Bingham, N., Goldie, C. and Teugels, J., Regular Variation, Encyclopedia of Mathematics and its Applications, Cambridge

University Press, Cambridge, UK, 1987.

Csorgo, S and Mason, D., Central limit theorems for sums of extreme values, Math. Proc. Camb. Phil. Soc. 98 (1985), $547-558$.

Davis, R. and Resnick, S., Tail estimates motivated by extreme value theory, Ann. Statist. 12 (1984), 1467-1487.

Geluk, J. and Haan, L. de, Regular Variation, Extensions and Tauberian Theorems, CWI Tract 40, Center for Mathematics and Computer Science, P.O. Box 4079, 1009 AB Amsterdam, The Netherlands, 1987.

Geluk, J., de Haan, L., Resnick, S., Stărică, C., Second order regular variation, convolution and the central limit theorem, Available from http://www.orie.cornell.edu/trlist/trlist.html as TR1133.ps.Z, Preprint (1995).

Goldie, C. and Smith, R., Slow variation with remainder: Theory and applications, Quarterly Journal of Mathematics $\mathbf{3 8}$ $(1987), 45-71$. 
Haan, L. de, Equivalence classes of regularly varying functions, Stochastic Processes and their Applications 2 (1974), 243-259.

Haan, L. de and Resnick, S., Estimating the limit distribution of multivariate extremes, Stochastic Models 9 (1993), 275-309. Hausler, E. and Teugels, J., On asymptotic normality of Hill's estimator for the exponent of regular variation, Ann. Statistics 13 (1985), 743-756.

Mason, D., Laws of large numbers for sums of extreme values, Ann. Probability 10 (1982), 754-764.

Mason, D., A strong invariance theorem for the tail empirical process, Ann. Inst. Henri Poincaré 24 (1988), $491-506$.

Resnick, S. and Stărică, C., Smoothing the Hill estimator, To appear: J. Applied Probability (1995).

Vervaat, W., Functional central limit theorems for processes with positive drift and their inverses, Z. Wahrscheinlichkeitstheory verw. Geb. 23 (1972), 249-253.

Laurens de Haan, Econometric Institute, Erasmus University Rotterdam, Po Box 1738, 3000 DR Rotterdam, The Netherlands

E-mail: dehaan@cs.few.eur.nl

Sidney Resnick, School of Operations Research and Industrial Engineering, Cornell University Ithaca, New YORK 14853-7501

E-mail: sid@orie.cornell.edu 